\title{
7 Schlussbetrachtung
}

Gegenstand der vorliegenden Untersuchung waren lexikalisch lizensierte Komplementweglassungen, d. h. solche, die durch die Verbwahl beschränkt sind. Die syntaktische Weglassung der Komplemente führt dabei nicht automatisch auch $\mathrm{zu}$ ihrer semantischen Tilgung. Vielmehr bleiben die entsprechenden semantischen Argumente in der Bedeutung des Satzes in einer unterspezifizierten Form präsent. Die Art, wie ein solches implizites Argument interpretiert werden muss, wird dabei aber nicht allein der Verwendungssituation, in der die Verben gebraucht werden, überlassen, sondern sie unterliegt einer Reihe von lexikalischen Einschränkungen. Auf der Grundlage der Literatur wurde dazu eine Arbeitshypothese formuliert (vgl. (13) und (295), hier erneut (444)), nach der unterschieden werden muss zwischen Weglassungen, die sich in erster Linie auf die Interpretation der in der Bedeutungsstruktur weiterhin vorhandenen Argumentstelle auswirken, vgl. (444a), und solchen, die zu einer Veränderung der Art des Ereignisbezugs führen, vgl. (444b):

(444) Interpretationsbesonderheiten bei Nicht-Spezifizierung einer Komplementstelle

a. Interpretationsbesonderheiten der impliziten Argumente:

i. Definitheit/Indefinitheit

ii. Semantische Spezialisierung

a) sortale Spezifikation

b) implizite Reflexivität

c) Polarisierung

d) Inklusion des Subjektkomplements

b. Besonderheiten des Ereignisbezugs:

i. Eventivität

ii. Eigenschaftszuschreibung

In der vorliegenden Arbeit wurden ausschließlich Komplementweglassungen untersucht, die Einfluss auf die Interpretation der impliziten Argumente zu haben scheinen, vgl. (444a). Weglassungen, die sich in erster Linie auf die Art des Ereignisbezugs auswirken, vgl. (444b), blieben hingegen unberücksichtigt.

Ziel der Untersuchung war es herauszufinden, inwieweit die in der Literatur beschriebenen Parameter zur lexikalischen Beschreibung impliziter Argumente ausreichen, um alle beobachtbaren semantischen Konsequenzen bei NichtSpezifizierung einer Argumentstelle zu erfassen. Vor dem Hintergrund, dass die Unterscheidung zwischen definiten und indefiniten impliziten Argumenten in 
der Literatur immer wieder Gegenstand kontroverser Diskussionen war und ist, standen hier Untersuchungen zu den semantischen Spezialisierungen impliziter Argumente im Vordergrund (vgl. Kapitel 5).

Eigene Voruntersuchungen gaben in diesem Zusammenhang Anlass zu der Vermutung, dass weitere Differenzierungen notwendig sind, um den Referenzmodus impliziter Argumente hinreichend erfassen und beschreiben zu können. In diesem Sinne wird folgende Forschungsfrage formuliert:

F1 Lassen sich neben den in der Literatur genannten Parametern - sortale Spezifikation, implizite Reflexivität und Polarisierung - weitere Kriterien zur lexikalischen Beschreibung semantischer Spezialisierungen impliziter Argumente ermitteln und wenn ja, welche?

Wurden in der Literatur Aussagen zur Argumentweglassung im Allgemeinen sowie zu möglichen semantischen Konsequenzen impliziter Argumente im Besonderen bislang zumeist ausgehend von der Annahme ,1 Verb = 1 Valenzträger' getroffen, wurde in der vorliegenden Arbeit die Auffassung vertreten, dass es eigentlich Verblesarten sind, von denen man annehmen kann, dass sie ihre Ergänzungen nach Zahl und Art bestimmen.

Unter der Prämisse, dass sich die einzelnen Verblesarten durch spezifische semantische und syntaktische Valenzrahmen sowie unterschiedliche Komplementweglassungsbedingungen auszeichnen, stellte sich die Frage, inwieweit die im Sinne von F1 beobachtbaren semantischen Spezialisierungen impliziter Argumente tatsächlich das Resultat von Argumentweglassung darstellen. Denkbar ist auch, dass sie auf die unterschiedlichen Verblesarten zurückzuführen sind. In diesem Sinne wird ergänzend folgende Forschungsfrage formuliert:

F2 Ist die einer Weglassungsoption entsprechende stellenreduzierte Alternativvalenz tatsächlich einer Verblesart zuzuordnen, der sie im Vergleich zu der nicht-stellenreduzierten Valenz derselben Lesart Interpretationsbesonderheiten hinzufügt, oder ist sie einer bestimmten von mehreren Verblesarten zuzuordnen, der sie keine Interpretationsbesonderheiten im Vergleich zu der nicht-stellenreduzierten Valenz hinzufügt?

Zur Beantwortung der beiden Fragen F1 und F2 wurden zunächst in einem theoretisch-reflektierenden Teil das hier zugrundegelegte multidimensionale Valenzkonzept sowie die damit einhergehende und hier vertretene Analyse fakultativer Ergänzungen dargestellt (Kapitel 2). Außerdem wurden Überlegungen zur Notwendigkeit einer Lesartenunterscheidung und den Möglichkeiten der Ermittlung 
von Verwendungsweisen sprachlicher Ausdrücke angestellt (Kapitel 3 und 4). Daran schlossen sich in einem praktisch-analytischen Teil umfangreiche Einzelverbuntersuchungen zu den möglichen semantischen Spezialisierungen impliziter Argumente an. Deren Organisation ergab sich aus der in (444) wiederholten Systematik zu den Interpretationsbesonderheiten impliziter Argumente. Entsprechend wurden Verben untersucht, die sortal spezifische implizite Argumente (Kapitel 5.1), implizit reflexive Argumente (Kapitel 5.2) und polarisierende implizite Argumente (Kapitel 5.4) aufweisen. Ausgehend von eigenen, im Vorfeld durchgeführten Untersuchungen wurden im Sinne von F1 zusätzlich Verben miteinbezogen, die bei Komplementweglassung eine Art Subjektinklusion erkennbar werden lassen (Kapitel 5.3).

In umfangreichen einzelverbbezogenen Überprüfungen, für die aufgrund der Spezifika des Untersuchungsgegenstandes keine strikt einheitliche Vorgehensweise möglich war, wurden insgesamt dreizehn Verben analysiert. Bei elf von ihnen ergab sich bereits vor Komplementweglassung eine klar erkennbare Lesartendifferenzierung. Legt man diese zugrunde, zeigte sich, dass es sich bei den in der Literatur beschriebenen sowie in eigenen Voruntersuchungen ermittelten semantischen Spezialisierungen impliziter Argumente (vgl. F1) untersuchungsgruppenübergreifend nicht um Interpretationsbesonderheiten stellenreduzierter Alternativvalenzen im Vergleich zu den nicht-stellenreduzierten Valenzen derselben Lesarten handelt. Vielmehr sind die stellenreduzierten Alternativvalenzen einer von mehreren Lesarten zuzuordnen. Diesen Verwendungsweisen fügen die stellenreduzierten Alternativvalenzen dann keine Interpretationsbesonderheiten im Vergleich zu den nicht-stellenreduzierten Valenzen hinzu, sondern sie übernehmen die sortalen Besonderheiten der jeweiligen Verblesart; die Weglassungen sind somit sortal-semantisch neutral.

Eine solche Analyse wurde in der vorliegenden Arbeit auch für die beiden Verben duschen und riechen vorgenommen. Bei ihnen fiel das Ergebnis der durchgeführten Lesartendifferenzierung allerdings nicht eindeutig aus. Entsprechend konnte bei diesen beiden Verben die in der Literatur vertretene Auffassung, wonach die einer Weglassungsoption entsprechende stellenreduzierte Alternativvalenz einer (zentralen) Lesart zuzuordnen ist, der sie im Vergleich zu der nicht-stellenreduzierten Valenz derselben Lesart Interpretationsbesonderheiten hinzufügt, nicht vollständig widerlegt werden.

Ausgehend davon, dass bei polysemen Verben mehrere voneinander zu unterscheidende Valenzträger vorliegen, haben die hier durchgeführten Untersuchungen mit Blick auf die in F1 und F2 formulierten Forschungsfragen somit ergeben, dass die in der Literatur genannten sowie in eigenen Voruntersuchungen ermittelten Parameter zur lexikalischen Beschreibung semantischer Spezialisierungen impliziter Argumente - sortale Spezifikation, implizite Reflexivität, Subjektinklusion und Polarisierung - zur Beschreibung beobachtbarer semantischer Effekte 
bei Komplementweglassung nicht benötigt werden. Sie sind nämlich nicht das Resultat von Weglassungen, sondern auf das Vorliegen unterschiedlicher Lesarten mit spezifischen semantischen und syntaktischen Valenzforderungen und eigenen Weglassungsbedingungen bereits vor Weglassung zurückzuführen.

In der Rückschau scheint dies auch durch die ausschließliche Fokussierung hier als polysem verstandener Verben und die fehlende Berücksichtigung von als homonym auszuweisender Verben unter diesem Aspekt bestätigt zu werden (vgl. auch die Anmerkungen zur Auswahl des Untersuchungsgegenstandes in Kapitel 1.2).

In Kapitel 6 wurden die Hauptuntersuchung ergänzende Überlegungen zu den in der Literatur beschriebenen definiten und indefiniten bzw. definitheitsneutralen impliziten Argumenten angestellt. Dabei wurde vor dem Hintergrund, dass insbesondere die Annahme indefiniter bzw. definitheitsneutraler impliziter Argumente immer wieder Gegenstand kritischer Diskussionen ist, geprüft, inwieweit sich die Ergebnisse der in Kapitel 5 durchgeführten Analysen zu den semantischen Spezialisierungen impliziter Argumente auch auf diesen Bereich übertragen lassen.

Ausgehend von den Beobachtungen in Kapitel 6 wurde folgende Hypothese entwickelt: Analog zu den zuvor dargestellten semantischen Spezialisierungen impliziter Argumente sind auch die in der Literatur als indefinit bzw. definitheitsneutral diskutierten Weglassungen nicht als Resultat von Komplementweglassungen zu interpretieren. Vielmehr sind die beobachtbaren Effekte auch hier auf das Vorliegen unterschiedlicher Lesarten bereits vor Komplementweglassung zurückzuführen. Die verschiedenen Valenzträger sind dabei mit unterschiedlichen Perspektivierungen verbunden: So kann differenziert werden zwischen einer transitiven Lesart, die das, was gegessen, getrunken oder gelesen wird bzw. wer geheiratet oder sich verlobt hat fokussieren, und einer intransitiven Lesart, die die vom Prädikat benannte Handlungsform als solche beschreibt. Die Annahme, dass es sich bei intransitiven Verbverwendungen tatsächlich um eine intransitive Lesart handelt, und nicht - wie häufig, u. a. unter dem Stichwort der Detransitivierung, angenommen - um eine valenzreduzierte Form der transitiven Verblesart, wird u.a. durch die fehlende definite Referenz eines nicht realisierten Themas bei entsprechenden intransitiven Verbverwendungen gestärkt (vgl. Kapitel 6.1 und 6.2).

Im Unterschied dazu sind definite Interpretationen impliziter Argumente nicht auf das Vorliegen unterschiedlicher Lesarten zurückzuführen. Vielmehr sind sie tatsächlich als Resultate von Komplementweglassung zu interpretieren. Weglassungen dieser Art zeichnen sich dadurch aus, dass entsprechende Verben in ihrer transitiven wie intransitiven Verwendung telische Ereignisse beschreiben. Bei intransitiven Verwendungen nimmt das nicht realisierte Thema definite 
Referenz an; entsprechend steht eine Argumentposition für das nicht-realisierte Thema in der lexikalisch-semantischen Repräsentation der Fakultativitätsvariante zur Verfügung.

Im Ergebnis der hier durchgeführten Untersuchungen lässt sich festhalten, dass einzig die Festlegung auf kontextuell gegebene Argumente bei definiter Weglassung als Effekt von lexikalisch bedingter Weglassung analysiert werden kann. Alle anderen beobachtbaren semantischen Besonderheiten impliziter Argumente ergeben sich aus den reichhaltigen Polysemiestrukturen der untersuchten Verben.

In diesem Sinne muss die eingangs als Arbeitshypothese entwickelte Systematik der Interpretationsbesonderheiten bei Nicht-Spezifizierung einer Komplementstelle folgendermaßen angepasst werden:

(445) Interpretationsbesonderheiten bei Nicht-Spezifizierung einer Komplementstelle

a. Interpretationsbesonderheiten der impliziten Argumente:

Definitheit

b. Besonderheiten des Ereignisbezugs:

i. Eventivität

ii. Eigenschaftszuschreibung

Da es sich bei den hier durchgeführten Untersuchungen um einzelverbbezogene Analysen (Kapitel 5) bzw. verbübergreifende Überlegungen zur Unterscheidung definiter und indefiniter bzw. definitheitsneutraler impliziter Argumente (Kapitel 6) handelt, sind aus den hier gemachten Beobachtungen keine Verallgemeinerungen $\mathrm{zu}$ ziehen. Vielmehr müssen weitere Untersuchungen $\mathrm{zu}$ anderen hier nicht überprüften Verben folgen. Dabei ist auch zu berücksichtigen, dass es sich bei Komplementweglassung häufig um ein gesprochensprachliches Phänomen handelt; ein Umstand, der aufgrund der (noch) unzureichenden Datengrundlage für gesprochenes Deutsch in der vorliegenden Arbeit weitestgehend unberücksichtigt blieb (vgl. Kapitel 1.3). Hierauf sollte bei nachfolgenden Untersuchungen stärker eingegangen werden.

Ebenfalls lohnenswert kann in diesem Zusammenhang ein Blick auf metonymische und metaphorische Prozesse der Lesartenerweiterung und ihre Konsequenzen für Komplementweglassung sein. 